\title{
MICROMACHINED MAGNETICALLY RECONFIGURABLE FREQUENCY-SELECTABLE SURFACES
}

\author{
Joe Zendejas, John Gianvittorio, Bongyoung Yoo, Yahya Rahmat-Samii, Ken Nobe, and Jack W. Judy \\ Electrical Engineering Department, University of California, Los Angeles \\ Los Angeles, CA, 90095 USA
}

\begin{abstract}
Reconfigurable frequency-selective surfaces have been realized by integrating an array of batch fabricated magnetic microactuators into an array of conductive dipole elements. The microactuators in the array consist of a $896 \times 168 \times 30 \mu \mathrm{m}^{3}$ ferromagnetic plate made of $40 \mathrm{Co}-60 \mathrm{Ni}$ attached to a pair of $400 \times 30 \times 1 \mu \mathrm{m}^{3}$ polysilicon torsion bars. The remanent magnetization in the hard ferromagnetic material allows relatively small magnetic fields $(\sim 1750 \mathrm{~A} / \mathrm{m}$ or $22 \mathrm{Oe})$ to induce significant angular deflections $\left(\sim 45^{\circ}\right)$. By rotating the dipole elements from $0^{\circ}$ to more than $45^{\circ}$, the filtering response of the frequency-selective surface can be tuned over a range of $5 \mathrm{GHz}$ at frequencies over $60 \mathrm{GHz}$.
\end{abstract}

\section{INTRODUCTION}

The expanding needs of RF and mm-wave system designers are pushing the limits of MEMS development [1-2]. Switchable and tunable RF and mm-wave systems are of great interest because they offer the potential to expand the functionality and capability of high-frequency communication systems.

Frequency-selective surfaces (FSSs) are periodic structures in two dimensions that can provide frequency filtering to incoming electromagnetic waves (Figure 1a). A common FSS example is the mesh screen on the door of a microwave oven. The mesh screen blocks electromagnetic radiation from the inside of the microwave oven while allowing the operator to see inside safely. Although FSSs have long been studied, they have experienced tremendous growth in the last ten years with new applications emerging (e.g., frequency filters or diplexers in high performance reflector antenna systems, advanced radome designs, and smart surfaces for stealth applications). These FSSs are typically designed with static elements for a specific frequency response (Figure 1b). The size and spacing of the periodic cells in an array of elements controls the overall frequency response and bandwidth of the surface (Figure 2) [3].

Although prior work on FSSs has only been on arrangements of static in-plane elements [4], in many applications it would be highly advantageous to adaptively reconfigure the frequency and bandwidth response of an FSS. However, a fundamental challenge has been achieving significant high-speed reconfigurability without compromising filtering performance. The frequency and bandwidth response of FSSs can be adjusted by reconfiguring the size, spacing, or orientation of the elements in the array.

Simulations performed using two methods developed at UCLA (i.e., a periodic moment method analysis technique and a finite difference time domain technique FDTD), have shown that the frequency response of an FSS is a strong function of dipole element tilt [5-7]. Rotating conductive elements in an array through an angle of $0^{\circ}$ to $30^{\circ}$, can significantly shift its overall frequency response. To enable the efficient control of mm-wave signals, single devices or arrays of microactuators are needed that can travel relatively large distances $(\sim 1 \mathrm{~mm})$ with a high degree of control and reliability.

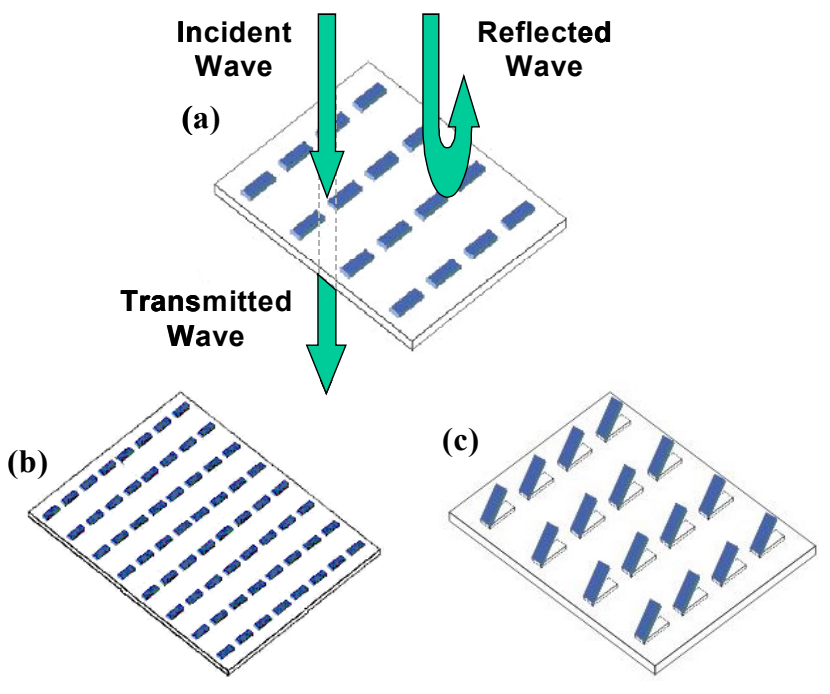

Figure 1. Frequency selective surfaces: (a) incident, reflected, and transmitted waves through an FSS; (b) an FSS with a different frequency response; (c) an FSS with rotating dipoles.

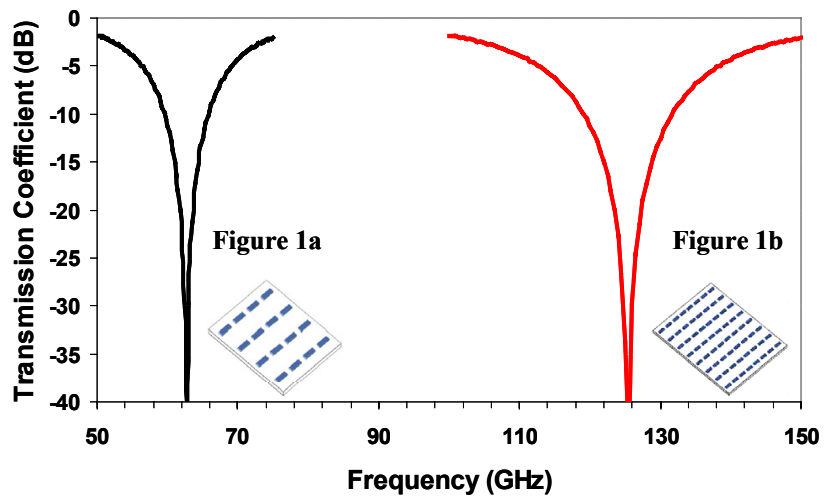

Figure 2. Simulated frequency responses of different static arrays as a function of element size and periodicity.

\section{ANALYSIS}

An attempt to look at the effect of a non-planar FSS was performed in [4]. In that work, the authors investigated a discrete number of non-planar configurations (of the periodic scatterer). Rigorous simulations in [5] have been used to show the effects of having the periodic elements rotated such that they are no longer confined to the periodic plane. An illustration of the concept of rotated dipoles is shown in Figure 1c. Even though the dipoles are tilted, the reflected field remains normal to the $x-y$ plane because the reflected wave is the fundamental Floquet mode, which depends on the angle of incidence, not the orientation of the periodic dipole. 


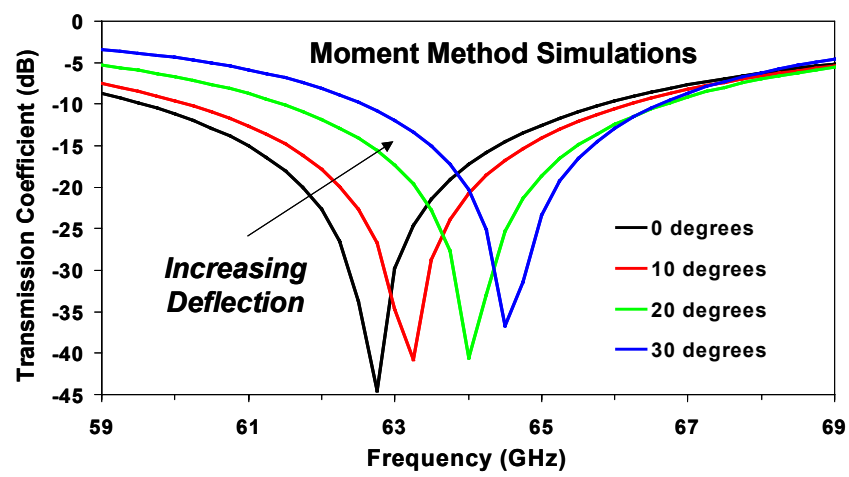

Figure 3. Simulations of the effect of angular deflection on the frequency response of an array of dipoles $\left(896 \times 168 \mu \mathrm{m}^{3}\right)$. Note the prominent shift upwards and to the right with increasing angular deflection.

Figure 3 shows the normal incidence transmission coefficient of an FSS composed of thin dipoles, as a function of dipole rotation $\left(\varphi=0^{\circ}\right.$ to $\left.30^{\circ}\right)$. The $\varphi=0^{\circ}$ curve shows a resonance when $\lambda / 2$ is approximately the length of the dipole element. Since the incident field is normal to the $x-y$ plane, in the limit $\varphi=90^{\circ}$ (i.e., dipoles parallel to the $z$-axis) no currents can be excited on the dipoles. An observation from Figure 3 is that the resonant frequency is shifted as the dipole tilt is increased. The shift in the resonant frequency is due to the mutual coupling between the dipoles. Since in this case the length of each dipole is $80 \%$ of the periodicity, the spacing between the dipoles is small. As the dipoles are tilted, the spacing between them increases, which changes the coupling. The change in the mutual coupling shifts the $\lambda / 2$ resonance.

\section{DESIGN}

Advances and developments in micromachining and MEMS technology can be used to fabricate an FSS array with elements capable of rotation, thereby enabling the tuning of the frequency response. Particularly well suited for this application is a ferromagnetic microactuator consisting of a ferromagnetic plate and non-magnetic torsional flexures (Figure 4). The device is actuated by an off-chip source for the magnetic field. The microactuation principle is essentially that of a compass needle the integrated magnetic microelements rotate with the applied magnetic field.

This microactuation technology combines the excellent mechanical robustness of polysilicon flexures with the large forces that can be generated by ferromagnetic materials in a magnetic field. The ability of microactuators made with technology to rotate through large out-of-plane deflections $\left(>90^{\circ}\right)$ has been previously demonstrated [9-10]. Accurate models for the static and dynamic behavior of this microactuation technology have also been developed and reveal that high-speed tuning of the dipole array is feasible [8-10].

The unique features that make this microactuator technology well suited for this application include: (1) no individual interconnects that might otherwise interfere with the frequency response and bandwidth of the surface are needed, and (2) dense arrays of actuators can all be driven simultaneously to the same angular deflection by changing only one parameter - the driving magnetic field (Figure 5).

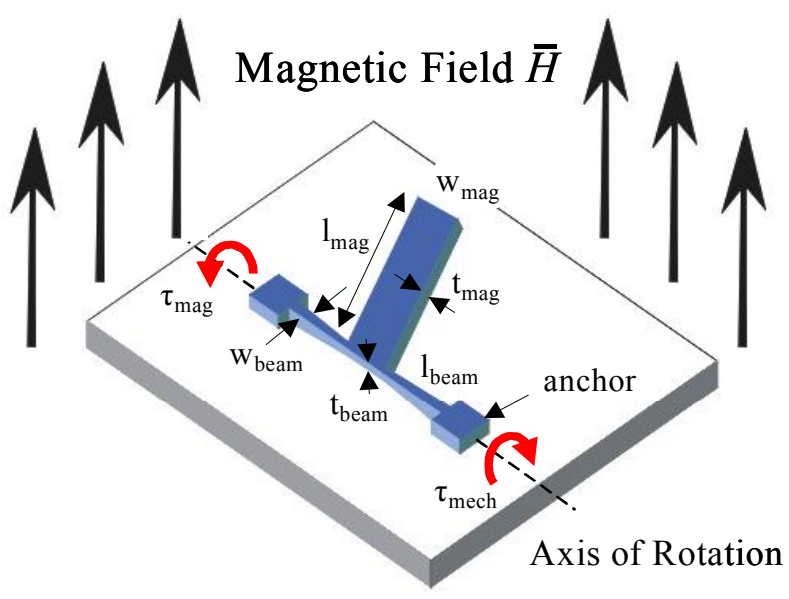

Figure 4. Ferromagnetic microactuators with torsional flexure driven by an off-chip source of magnetic field.

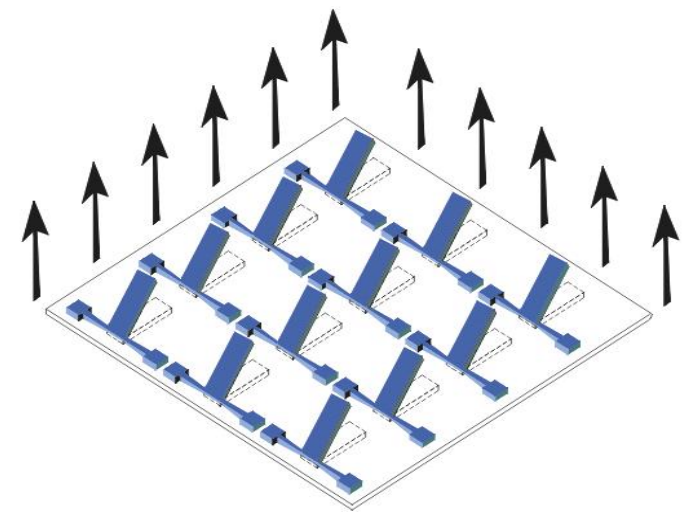

Figure 5. Array of actuators being driven by a magnetic field.

As shown in [10], the appropriate material for this microactuator technology is a hard magnetic material (i.e., one with a large coercivity and remanent magnetization). In this work we have developed a process for electroplating $40 \mathrm{Co}-60 \mathrm{~N}$ as the ferromagnetic material with the magnetic properties described in Figure 6, with coercivity $6000 \mathrm{~A} / \mathrm{m}$ (75 Oe) and magnetic saturation $1.0 \mathrm{~T}$.

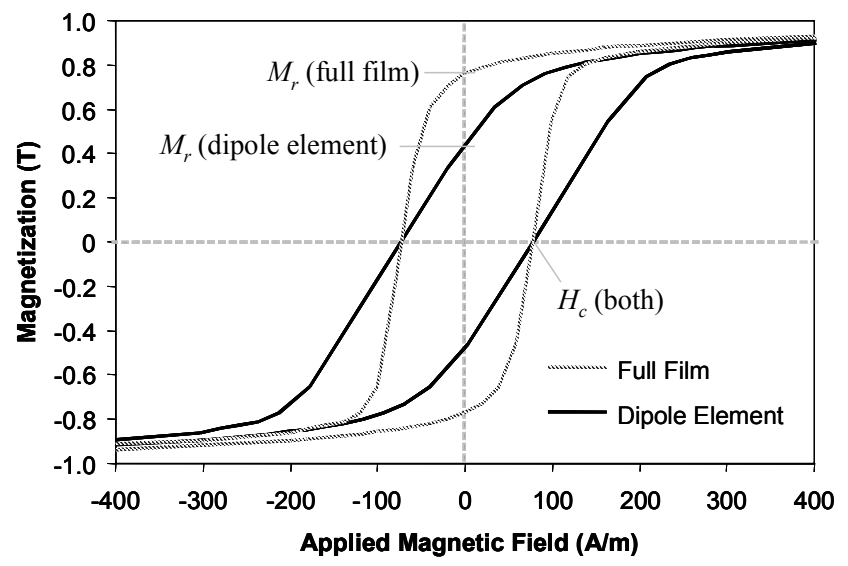

Figure 6. Measured B-H loop for 30- $\mu$ m-thick films of electroplated 40Co-60Ni with no demagnetizing field (dashed) and with the effects of a calculated demagnetization field from a $896 \times 168 \times 30 \mu^{3}$ element (solid). 
Since the FSS was designed with an array of $896 \times 168 \mu \mathrm{m}^{2}$ elements, the demagnetization coefficients are $N_{a}=0.012$, $N_{b}=0.14$, and $N_{c}=0.835$ (i.e., assuming a magnetic film thickness of $30 \mu \mathrm{m}$ ) [9]. The result being that the $B-H$ loop of an individual microactuator element (i.e., solid line in Figure 6) will be sheared relative to the $B-H$ loop measured in a sample with negligible demagnetization coefficients in the plane of the sample (i.e., $N_{a} \approx 0, N_{b} \approx 0$, and $N_{c} \approx 1$ and illustrated with the dashed line in Figure 6). Thus the maximum remanent magnetization in an individual element will be $\sim 0.45 \mathrm{~T}$. If the torsional flexure is made of polysilicon and has the following dimensions $400 \times 30 \times 1 \mu \mathrm{m}^{3}$, the mechanical deflection as a function of magnetic field directed normal to the surface of the wafer is given in Figure 7. The magnetic drive field required for a $45^{\circ}$ deflection is only $1750 \mathrm{~A} / \mathrm{m}$ (22 Oe), as compared to $7000 \mathrm{~A} / \mathrm{m}(88 \mathrm{Oe})$ in previous work [9-10]. The much higher remanent magnetization of the $40 \mathrm{Co}-60 \mathrm{Ni}$ alloys provides a tremendous boost in performance that allows for smaller driving fields and low-power operation.

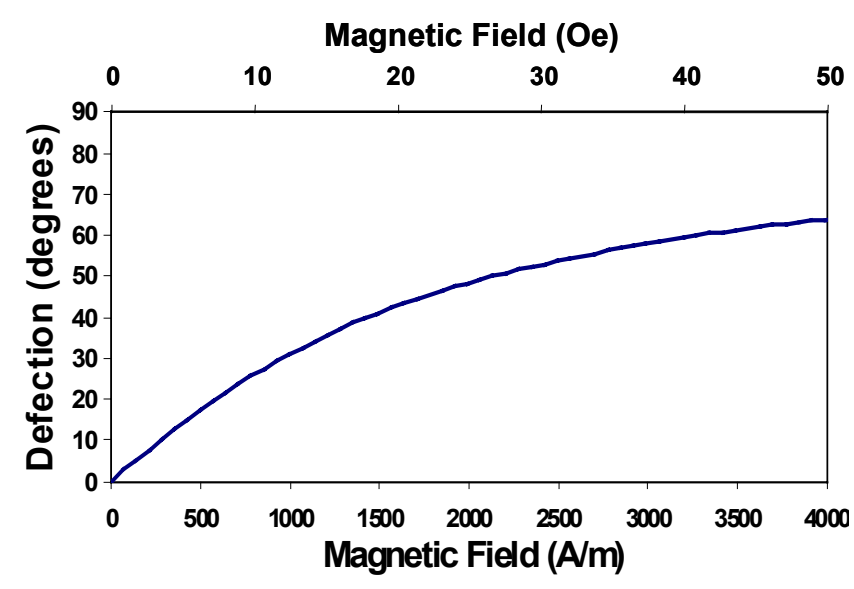

Figure 7. Theoretical angular deflection of a $896 \times 168 \times 30 \mu^{3}$ $40 \mathrm{Co}-60 \mathrm{Ni}$ plate attached to a pair of $400 \times 30 \times 1 \mu^{3}$ polysilicon torsional beams.

\section{FABRICATION}

There are many important considerations when designing the fabrication process for a micromachined magnetically reconfigurable FSS: substrate loss, magnetic material, and process integration. In order to achieve the highest Q response, a low-loss substrate is required that minimizes the amount of attenuation contributed from the substrate. In this work, a $<100>$ p-type silicon wafer with 30-60 $\Omega$-cm was used. To achieve a large angle of deflection for a low external magnetic field, a thick film of hard magnetic material with substantial remanent magnetization is needed.

The fabrication process starts for devices reported here with the deposition of a 2- $\mu$ m-thick layer of low-temperature oxide (LTO). Anchors for the torsion beam pads are then patterned into the LTO sacrificial layer. A 1- $\mu$ m-thick film of undoped polysilicon is then deposited using low-pressure chemical-vapor deposition (LPCVD). The mechanical structures are then patterned into the polysilicon (Figure 8a). An adhesion layer of chrome and a seed layer of nickel are evaporated onto the polysilicon plates. Then a $0.2-\mu \mathrm{m}$-thick layer of titanium is evaporated onto the entire surface to act as the conduction layer between elements during plating. A $48-\mu \mathrm{m}$-thick photoresist mold (Shipley, STR 1045) is then patterned on the Ti conduction layer and around the $\mathrm{Cr}-\mathrm{Ni}$ seed layer where the electroplated magnetic material is deposited (Figure $8 \mathrm{~b}$ ). The Co-Ni alloy is electroplated from a chloride bath with dc plating. The stress in the deposit is a minimum at an alloy composition of $40 \mathrm{Co}-60 \mathrm{Ni}$ when saccharin is added as a stress reducer. A 1- $\mu \mathrm{m}$-thick layer of $\mathrm{Au}$ is deposited to add a high conductivity surface to the dipole element. After plating the photoresist plating mold is stripped (Figure 8c) and the excess conduction layer is removed. The final release is performed in $49 \%$ HF for 60 min to release the microstructures, which are then dried by $\mathrm{CO}_{2}$-supercritical drying to complete the fabrication process (Figure $8 \mathrm{~d}$ ). An image of a typical microfabricated dipole element is given in Figure 9.
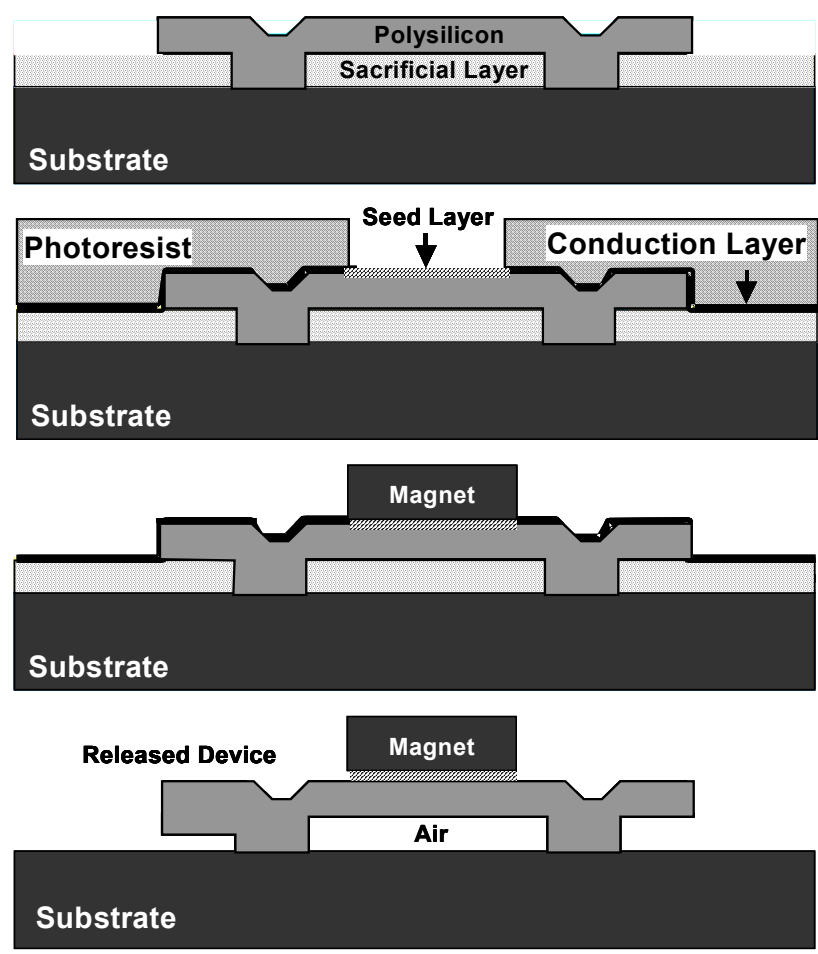

Figure 8. Fabrication process for FSS elements: (a) deposition and etching of sacrificial and microflexure layers; (b) deposition of adhesion, seed, and conduction layers with formation of plating mold; (c) plated Co-Ni alloy and stripped plating mold; (d) removal of seed layer and the sacrificial layer to release the device.

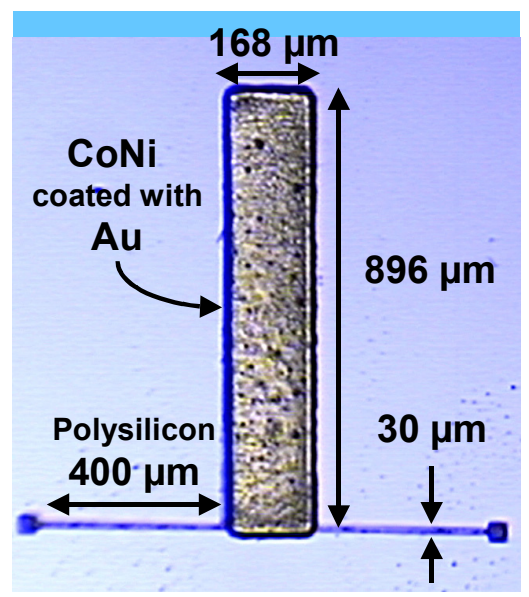

Figure 9. Image of a single dipole with a pair of $400 \times 30 \times 1 \mathrm{\mu m}^{3}$ polysilicon torsion beams and a $896 \times 168 \times 31 \mu^{3}$ dipole. 


\section{TESTING}

The frequency response of an FSS, consisting of a $25 \times 25$ element array of $896 \times 168 \times 31 \mu \mathrm{m}^{3}$ elements made of $1 \mu \mathrm{m}$ of $\mathrm{Au}$ on $30 \mu \mathrm{m}$ of $40 \mathrm{Co}-60 \mathrm{Ni}$ on $1 \mu \mathrm{m}$ of undoped polysilicon (Figure 9), is measured using an HP8510 network analyzer with a millimeter-wave source setup (Figure 10). The FSS is uniformly illuminated with a horn antenna and microwave-absorbing material is used to decrease edge diffraction and noise. Experimental results of the effect of angular deflection on frequency response, reveals the trend predicted by simulations (i.e., the frequency shift upwards with increasing angle of deflection shown in Figure 3).

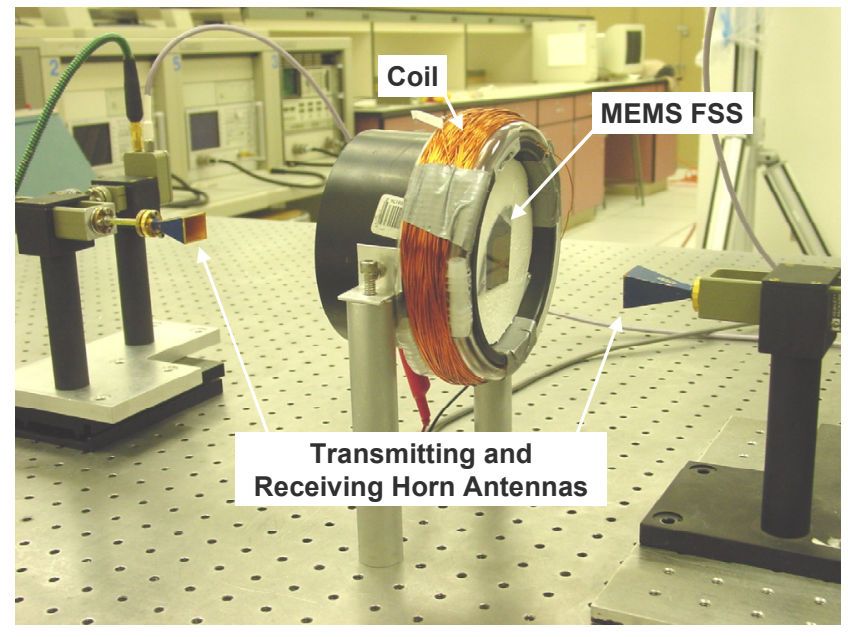

Figure 10. Experimental setup for characterizing the frequency response of the MEMS-enabled reconfigurable FSS.

From the measured results (Figure 11), it can be seen that as the elements are tilted, the blocked frequency band shifts higher. However, the rejection of the filter is gradually reduced.

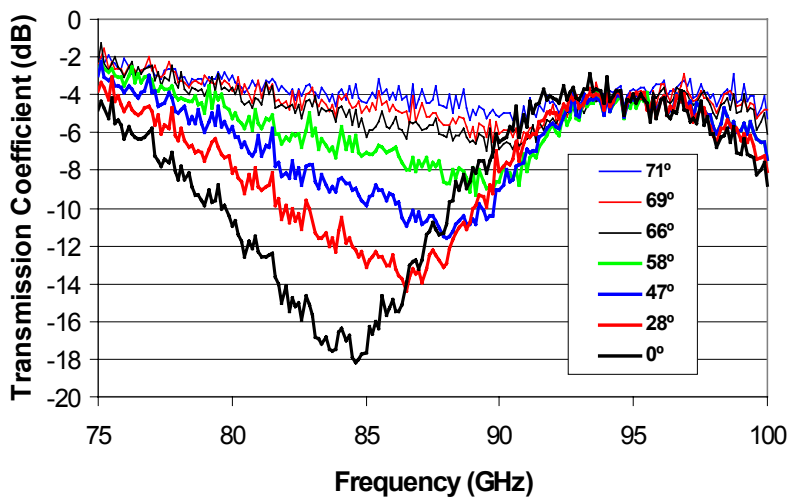

Figure 11. Measured frequency response of rotated MEMS FSS for normal incidence.

\section{CONCLUSIONS}

Rotating the dipole elements in a FSS can tune its frequency response at high frequencies (e.g., $>60 \mathrm{GHz}$ ) over a wide range $(\sim 5 \mathrm{GHz})$. Micromachining and MEMS technologies, specifically arrays of ferromagnetic microactuators with polysilicon torsional flexures, can be used to realize reconfigurable FSSs. Initial results indicate that substrate losses limit the $\mathrm{Q}$ of the filter response. Future implications of this work include new agile high-frequency communication systems and stealth technology.

\section{ACKNOWLEGMENT}

The authors would like to thank that staff of the UCLA Nanoelectronics Research Laboratory and the staff of the UCLA Center for High Frequency Research. This work was funded in part by NSF XYZ \#0089095 and an AT\&T Fellowship.

\section{REFERENCES}

[1] Clark C.-T. Nguyen, L. P. B. Katehi, G. M. Rebeiz, "Micromachined devices for wireless communications", Proceedings of the IEEE, vol. 86, no. 8, pp. 1756-1768.

[2] Clark C.-T. Nguyen, "Frequency-selective MEMS for miniaturized low-power communication devices", IEEE Transactions on Microwave Theory and Techniques, vol. 47, no. 8, pp. 1486-1503.

[3] Y. Rahmat-Samii and A. N. Tulintseff, "Diffraction analysis of frequency selective reflector antennas", IEEE Transactions Antennas Propagation, vol. AP-41, no. 4, pp. 476-487, 1993.

[4] B. Irvine, W. L. Ko, and R. Mittra, "A comparative study of angular scattering characteristics of a class of nonplanar frequency selective surfaces", Electromagnetics, vol. 15, no. 6, pp. 649-663, 1995

[5] Alon Barlevy and Yahya Rahmat-Samii, "Control of Resonant bandwidth in frequency selective surfaces by tilting the periodic elements", Microwave and Optical Technology Letters, vol. 21, no. 2, pp. 114-117, 1999.

[6] Alon Barlevy, and Yahya Rahmat-Samii. "Characterization of Electromagnetic Band-Gaps Composed of Multiple Periodic Tripods with Interconnecting Vias: Concept, Analysis, and Design." IEEE Transactions on Antennas and Propagation, vol. 49, no. 3, pp. 343-353, 2001.

[7] Yahya Rahmat-Samii and H. Mosallaei. "Electromagnetic Band-gap Structures: Classification, Characterization, and Applications." Proceedings of $11^{\text {th }}$ International Conference on Antennas and Propagation, April 2001, vol. 2, pp. 560-564.

[8] Jack W. Judy, Richard. S. Muller, and Hans. H. Zappe, "Magnetic microactuation of polysilicon flexure structures," IEEE Journal of Microelectromechanical Systems, vol. 4, no. 4, pp. 162-169, 1995.

[9] Jack W. Judy and Richard. S. Muller, "Magnetic microactuation of torsional polysilicon structures," Sensors and Actuators A (Physical), vol. A53, no. 1-4, pp. 392-396, 1996.

[10] Jack W. Judy and Richard. S. Muller, "Magnetically Actuated, Addressable Microstructures" IEEE Journal of Microelectromechanical Systems, vol. 6, no. 3, pp. 249-256, 1997. 